\title{
Synthesis of Organoplatinum Oligomers by Employing N-Donor Bridges with Predesigned Geometry: Structural and Photophysical Properties of Luminescent Cyclometalated Platinum(II) Macrocycles
}

\author{
Siu-Wai Lai, Michael C. W. Chan, Kung-Kai Cheung, Shie-Ming Peng, ${ }^{\dagger}$ and \\ Chi-Ming Che*
}

Department of Chemistry, The University of Hong Kong, Pokfulam Road, Hong Kong

Received May 10, 1999

\begin{abstract}
A series of luminescent di- and trimeric cyclometalated platinum(II ) macrocycles, namely, $[\mathrm{Pt}(\mathrm{C}-\mathrm{N})(\mathrm{N}-\mathrm{N})]_{2}(\mathrm{~N}-\mathrm{N}=$ pyrazolate, pz (2); 7-azaindolate, aza (3); $\mathrm{C}-\mathrm{N}=2$-(2'-thienyl)pyridyl, Thpy (a); 7,8-benzoquinolate, Bzqn (b); 2-phenylpyridyl, Phpy (c)) and [Pt(C-N)$(\mathrm{N}-\mathrm{N})]_{3}(\mathrm{~N}-\mathrm{N}=$ benzimidazolate, bzim (4); $\mathrm{C}-\mathrm{N}=$ Thpy (a) and Bzqn (b)), are synthesized in high yields (70-90\%). The rigid, predefined coordination geometry of the pz, aza, and bzim bridging ligands ensure the efficient and selective assembly of the respective cyclic oligomers. The stacking arrangements in the crystal structures of $\mathbf{1 a}$ and $\mathbf{4 a}$ reveal $\pi-\pi$ interactions between 2-(2'-thienyl)pyridyl moieties. The lowest energy absorption band in the UV - vis spectra is assigned to metal-to-ligand charge transfer (MLCT) transitions. The $298 \mathrm{~K}$ fluid emissions of complexes 2-4a bearing Thpy ligands are assigned to transitions with mixed MLCT and IL (intraligand) character. In crystalline form, the observed redshift is attributed to excimeric emission arising from $\pi$-stacking in the sol id state. In contrast, a number of Bzqn derivatives are nonemissive in $\mathrm{CH}_{2} \mathrm{Cl}_{2}$ at $298 \mathrm{~K}$. The energy of the lowest energy UV-vis and emissive bands for Thpy oligomers are red-shifted relative to the Bzqn and Phpy congeners. Significantly, Thpy complexes and especially the $\mu$-pyrazolate species $2 \mathbf{a}\left(\tau_{0}=15.5 \mu \mathrm{s}, \phi_{0}=0.18\right)$ emit with longer lifetimes and higher quantum yields.
\end{abstract}

\section{Introduction}

Multidentate nitrogen-donor ligands are extensively utilized for the assembly of cyclic supermolecules. Pyrazolyl, 1,2 imidazolyl, ${ }^{3}$ and particularly pyridyl ${ }^{4-7}$ derivatives have been incorporated into the design and construction of novel oligometallic macrocycles and cage

\footnotetext{
* Corresponding author. Fax: +852 2857 1586. E-mail: cmche@ hkucc.hku.hk.

† Department of Chemistry, National Taiwan University, Taipei, Taiwan.

(1) Reviews: (a) Trofimenko, S. Prog. Inorg. Chem. 1986, 34, 115. (b) La Monica, G.; Ardizzoia, G. A. Prog. Inorg. Chem. 1997, 46, 151. (2) Pt examples: (a) Minghetti, G.; Banditelli, G.; Bonati, F. J . Chem. Soc., Dalton Trans. 1979, 1851. (b) Goel, A. B.; Goel. S.; Vanderveer, D. Inorg. Chim. Acta 1984, 82, L9. (c) J ain, V. K.; Kannan, S.; Tiekink, E. R. T. J. Chem. Soc., Dalton Trans. 1993, 3625. (d) Stobart, S. R.; Dixon, K. R.; Eadie, D. T.; Atwood, J . L.; Zaworotko, M. D. Angew. Chem., Int. Ed. Engl. 1980, 19, 931. Other metals: (e) Chong, K. S.; Rettig, S. J .; Storr, A.; Trotter, J . Can. J . Chem. 1979, 57, 3090. (f) Murray, H. H.; Raptis, R. G.; Fackler, J. P., J r. Inorg Chem. 1988, 27, 26. (g) Pinillos, M. T.; Tejel, C.; Oro, L. A.; Apreda, M. C.; Foces-Foces, C.; Cano, F. H. J . Chem. Soc., Dalton. Trans. 1989, 1133. (h) J effery, J . C.; J ones, P. L.; Mann, K. L. V.; Psillakis, E.; McCleverty, J . A.; Ward, M. D.; White, C. M. Chem. Commun. 1997, 175.

(3) (a) Tiripicchio, A.; Tiripicchio Camellini, M.; Usón, R.; Oro, L. A.; Ciriano, M. A.; Pinillos, M. T. J . Organomet. Chem. 1982, 224, 207. (b) Chaudhuri, P.; Karpenstein, I.; Winter, M.; Butzlaff, C.; Bill, E.; Trautwein, A. X.; Flörke, U.; Haupt, H.-J.J. Chem. Soc., Chem. Commun. 1992, 321. (c) Rüttimann, S.; Bernardinelli, G.; Williams, A. F. Angew. Chem., Int. Ed. Engl. 1993, 32, 392. (d) Matsumoto, N.; Motoda, Y.; Matsuo, T.; Nakashima, T.; Re, N.; Dahan, F.; Tuchagues, J .-P. Inorg. Chem. 1999, 38, 1165. Related nucleobases: (e) Smith, D. P.; Baralt, E.; Morales, B.; Olmstead, M. M.; Maestre, M. F.; Fish, R. H. J . Am. Chem. Soc. 1992, 114, 10647. (f) Chen, H.; Olmstead, M. M.; Smith, D. P.; Maestre, M. F.; Fish, R. H. Angew. Chem., Int. Ed.
} Engl. 1995, 34, 1514. complexes. Nevertheless, integration of photoluminescent moieties into these potential molecular receptors is rarely undertaken, although this can confer chemicalsensing applications. ${ }^{8}$ In this context, cyclometalated platinum(II) complexes bearing substituted pyridyl and 2,2'-bipyridyl ligands are promising candidates because they display emissive metal-to-ligand charge transfer (MLCT) excited states in solution, ${ }^{9-14}$ and these emissions are extremely environment-sensitive. ${ }^{15,16}$

In this work, our intention was to fabricate platinumcontaining macrocycles by connecting cyclometalated Pt-

(4) (a) Fujita, M.; Oguro, D.; Miyazawa, M.; Oka, H.; Yamaguchi, K.; Ogura, K. Nature 1995, 378, 469. (b) Fujita, M. Chem. Soc. Rev. 1998, 27, 417.

(5) Stang, P. J .; Olenyuk, B. Acc. Chem. Res. 1997, 30, 502.

(6) Slone, R. V.; Benkstein, K. D.; Bélanger, S.; Hupp, J . T.; Guzei, I. A.; Rheingold, A. L. Coord. Chem. Rev. 1998, 171, 221.

(7) (a) Leung, W. H.; Cheng, J . Y. K.; Hun, T. S. M.; Che, C. M.; Wong, W. T.; Cheung, K. K. Organometallics 1996, 15, 1497. (b) Schnebeck, R.-D.; Randaccio, L.; Zangrando, E.; Lippert, B. Angew. Chem., Int. Ed. 1998, 37, 119. (c) Hall, J . R.; Loeb, S. L.; Shimizu, G. K. H.; Yap, G. P. A. Angew. Chem., Int. Ed. 1998, 37, 121. (d) Schneider, R.; Hosseini, M. W.; Planeix, J.-M.; De Cian, A.; Fischer, J . Chem. Commun. 1998, 1625.

(8) Slone, R. V.; Yoon, D. I.; Calhoun, R. M.; Hupp, J . T. J . Am Chem. Soc. 1995, 117, 11813.

(9) Chassot, L.; Müller, E.; von Zelewsky, A. I norg. Chem. 1984, 23, 4249

(10) Sandrini, D.; Maestri, M.; Balzani, V.; Chassot, L.; von Zelewsky, A. J. Am. Chem. Soc. 1987, 109, 7720.

(11) (a) Chan, C. W.; Lai, T. F.; Che, C. M.; Peng, S. M. J . Am. Chem. Soc. 1993, 115, 11245. (b) Chan, C. W.; Cheng, L. K.; Che, C. M. Coord Chem. Rev. 1994, 132, 87.

(12) (a) Cheung, T. C.; Cheung, K. K.; Peng, S. M.; Che, C. M. J . Chem. Soc., Dalton Trans. 1996, 1645. (b) Lai, S. W.; Chan, M. C. W.; Cheung, T. C.; Peng, S. M.; Che, C. M. Inorg. Chem., in press. 
(II) luminophores with various bidentate $\mathrm{N}$-donor linkers. The pz ( $\mathrm{Hpz}=$ pyrazole), aza (Haza = 7-azaindole), and bzim ( $\mathrm{Hbzim}=$ benzimidazole) ligands exhibit predefined yet different geometrical characteristics. Hence accurate design is anticipated to yield specific molecular architectures that are thermodynamically favored. A ligand-directed strategy for the synthesis of triplatinum macrocycles by employing bzim as a rigid nonlinear bridging motif has been communicated. ${ }^{17}$ We now describe the preparation, crystal structures, and photophysics of luminescent platinum(II) cyclic dimers (bearing pz and aza) and trimers (bearing bzim) plus their monomeric precursors. By varying the $\mathrm{N}$-donor bridges and the cyclometal ating ligands, namely, Thpy (HThpy = 2-(2'-thienyl)pyridine), Bzqn (HBzqn = 7,8benzoquinoline), and Phpy (HPhpy = 2-phenylpyridine), we are able to assess trends and changes in emissive properties. Seminal spectroscopic studies by Gray and co-workers on $d^{8}-d^{8}$ binuclear species containing $\mu$-pyrazol ate ligands have appeared. ${ }^{18,19}$

\section{Experimental Section}

General Procedures. The following chemicals were obtained from Aldrich Chemical $\mathrm{Co}$. and used as received: $\mathrm{K}_{2-}$ $\mathrm{PtCl}_{4}$ (98\%), 2-phenylpyridine (HPhpy), 7,8-benzoquinoline (HBzqn), sodium hydride, pyrazole $(\mathrm{Hpz})$, benzimidazole (H bzim), and 7-azaindole (H aza). 2-(2'-Thi enyl)pyridine (HThpy) was purchased from Lancaster Chemical Co. [ $\left.{ }^{n} \mathrm{Bu}_{4} \mathrm{~N}\right][\mathrm{Pt}-$ $(\mathrm{Phpy}) \mathrm{Cl}_{2}$ ] (1c) was prepared by literature methods. ${ }^{20}$ Synthe sis of $\mathbf{4 a}, \mathbf{b}$ has been described previously. ${ }^{17}$ Dichloromethane for photophysics studies was washed with concentrated sulfuric acid, 10\% sodium hydrogen carbonate, and water, dried by calcium chloride, and distilled over calcium hydride. All other solvents were purified according to conventional methods. ${ }^{21}$

Fast atom bombardment (FAB) mass spectra were obtained on a Finnigan Mat 95 mass spectrometer. ${ }^{1} \mathrm{H}(300 \mathrm{MHz})$ and ${ }^{13} \mathrm{C}(126 \mathrm{MHz})$ spectra were recorded on DPX 300 and 500 Bruker FT-NMR spectrometers, respectively, with chemical shifts (in ppm) relative to tetramethylsilane. Elemental analysis was performed by the Institute of Chemistry at the Chinese Academy of Sciences, Beijing. Infrared spectra were recorded as $\mathrm{KBr}$ plates on a BIO RAD FT-IR spectrometer. UV-vis spectra were recorded on a Perkin-EImer Lambda 19 UV/vis spectrophotometer.

Emission and Lifetime Measurements. Steady-state emission spectra were recorded on a SPEX 1681 Fluorolog-2 series F111Al spectrophotometer. Low-temperature (77 K) emission spectra for frozen and solid-state samples were recorded in $5 \mathrm{~mm}$ diameter quartz tubes, which were placed

(13) (a) Tse, M. C.; Cheung, K. K.; Chan, M. C. W.; Che, C. M. Chem. Commun. 1998, 2295. (b) Lai, S. W.; Chan, M. C. W.; Cheung, K. K.; Che, C. M. Organometallics, in press.

(14) (a) Zheng, G. Y.; Rillema, D. P.; DePriest, J .; Woods, C. Inorg. Chem. 1998, 37, 3588. (b) Zheng, G. Y.; Rillema, D. P. Inorg. Chem. 1998, 37, 1392

(15) Liu, H. Q.; Peng, S. M.; Che, C. M. J . Chem. Soc., Chem. Commun. 1995, 509.

(16) (a) Wu, L. Z.; Cheung, T. C.; Che, C. M.; Cheung, K. K.; Lam, M. H. W. Chem. Commun. 1998, 1127. (b) Wong, K. H.; Chan, M. C. W.; Che, C. M. Chem. Eur. J ., in press.

(17) Lai, S. W.; Chan, M. C. W.; Peng, S. M.; Che, C. M. Angew. Chem., Int. Ed. 1999, 38, 669.

(18) Marshall, J. L.; Stobart, S. R.; Gray, H. B. J . Am. Chem. Soc. 1984, 106, 3027

(19) Bailey, J . A.; Miskowski, V. M.; Gray, H. B. Inorg. Chem. 1993 $32,369$.

(20) Craig, C. A.; Garces, F. O.; Watts, R. J .; Palmans, R.; Frank, A. J. Coord. Chem. Rev. 1990, 97, 193.

(21) Perrin, D. D.; Armarego, W. L. F.; Perrin, D. R. Purification of Laboratory Chemicals, 2nd ed.; Pergamon: Oxford, 1980. in a liquid nitrogen Dewar equipped with quartz windows. Emission spectra were corrected for monochromator and photomultiplier efficiency and for xenon lamp stability. Details of emission quantum yield determinations by the method of Demas and $\mathrm{Crosby}^{22}$ have been given. ${ }^{11}$ Emission lifetime measurements were performed with a Quanta Ray DCR-3 pulsed Nd:YAG laser system (pulse output $355 \mathrm{~nm}, 8 \mathrm{~ns})$. The emission signals were detected by a Hamamatsu R928 photomultiplier tube and recorded on a Tektronix model 2430 digital oscilloscope. Errors for $\lambda$ values $( \pm 1 \mathrm{~nm}), \tau( \pm 10 \%)$, and $\phi( \pm 10 \%)$ are estimated.

Synthesis. [Pt(Thpy)(HThpy)CI], 1a. $\left[{ }^{\mathrm{B} B u_{4}} \mathrm{~N}_{2}\left[\mathrm{PtCl}_{4}\right]\right.$ was prepared in the organic phase of a phase-transfer metathesis reaction by mixing $\mathrm{K}_{2} \mathrm{PtCl}_{4}(0.20 \mathrm{~g}, 0.48 \mathrm{mmol})$ in $\mathrm{H}_{2} \mathrm{O}(10 \mathrm{~mL})$ with ${ }^{\mathrm{n}} \mathrm{Bu}_{4} \mathrm{NCl}(0.58 \mathrm{~g}, 2.09 \mathrm{mmol})$ in $\mathrm{CH}_{2} \mathrm{Cl}_{2}$ (30 mL). ${ }^{20} \mathrm{HThpy}$ $(0.32 \mathrm{~g}, 1.99 \mathrm{mmol})$ in $\mathrm{CH}_{3} \mathrm{OH}(30 \mathrm{~mL})$ was then added to the $\mathrm{CH}_{2} \mathrm{Cl}_{2}$ phase. The red solution was stirred at reflux for $48 \mathrm{~h}$ until an orange solution was obtained. Upon removal of solvent, the orange oily solid was washed with hexane $(3 \times$ $10 \mathrm{~mL})$ and cold acetone $(10 \mathrm{~mL})$. Recrystallization by diffusion of diethyl ether into $\mathrm{a} \mathrm{CH}_{2} \mathrm{Cl}_{2}$ solution afforded orange crystals: yield $0.20 \mathrm{~g}, 74 \%$. Anal. Calcd for $\mathrm{C}_{18} \mathrm{H}_{13} \mathrm{~N}_{2} \mathrm{~S}_{2} \mathrm{PtCl}$ : C, 39.17; H, 2.37; N, 5.08. Found: C, 39.25; H, 2.24; N, 5.04 . FAB-MS: $\mathrm{m} / \mathrm{z} 552\left[\mathrm{M}^{+}\right], 516\left[\mathrm{M}^{+}-\mathrm{Cl}\right]$. IR (Nujol): $v=1603$, $1549,1516 \mathrm{~cm}^{-1}$. ${ }^{1} \mathrm{H}$ NMR $\left(\mathrm{CD}_{2} \mathrm{Cl}_{2}\right): 6.07(\mathrm{~d}, 1 \mathrm{H}, \mathrm{J}=4.8 \mathrm{~Hz})$ $7.03(\mathrm{~m}, 2 \mathrm{H}), 7.22-7.33(\mathrm{~m}, 3 \mathrm{H}), 7.46(\mathrm{~d}, 1 \mathrm{H}, \mathrm{J}=5.0 \mathrm{~Hz})$ 7.71-7.98 (m, 4H), 9.15 (d with broad ${ }^{195} \mathrm{Pt}$ satellites, $1 \mathrm{H}$, 3) $\left.{ }_{\mathrm{HH}}=5.8 \mathrm{~Hz},{ }^{3} \mathrm{PtH}_{\mathrm{PtH}}=47 \mathrm{~Hz}\right), 9.41$ (d with broad ${ }^{195} \mathrm{Pt}$ satellites, $\left.1 \mathrm{H},{ }^{3} \mathrm{HH}_{\mathrm{HH}}=5.3 \mathrm{~Hz},{ }^{3} \mathrm{PtH}_{\mathrm{PtH}}=42 \mathrm{~Hz}\right) \cdot{ }^{13} \mathrm{C}\left\{{ }^{1} \mathrm{H}\right\} \mathrm{NMR}$ $\left(\mathrm{CD}_{2} \mathrm{Cl}_{2}\right): 117.5,119.8,124.0,126.5,127.4,128.4,129.7,130.1$, 130.4, 138.3, 139.8, 141.2, 145.4, 151.4, 155.1, 156.0, 163.4.

[Pt(Thpy)(pz) ] 2, 2a. A mixture of pyrazole $(0.03 \mathrm{~g}, 0.40$ $\mathrm{mmol}$ ) and excess $\mathrm{NaH}$ in THF (10 mL) under a $\mathrm{N}_{2}$ atmosphere was stirred for $30 \mathrm{~min}$ until the evolution of hydrogen ceased. The solution was filtered, added to $1 \mathbf{a}(0.20 \mathrm{~g}, 0.36 \mathrm{mmol})$ in $\mathrm{CH}_{2} \mathrm{Cl}_{2}(30 \mathrm{~mL})$, and stirred under reflux for $30 \mathrm{~h}$. The resultant orange solution was concentrated to $5 \mathrm{~mL}$, and addition of diethyl ether afforded an orange solid. Recrystallization by diffusion of diethyl ether into a dichloromethane solution afforded orange crystals: yield $0.11 \mathrm{~g}, 72 \%$. Anal. Calcd for $\mathrm{C}_{24} \mathrm{H}_{18} \mathrm{~N}_{6} \mathrm{~S}_{2} \mathrm{Pt}_{2}$ : C, 34.12; H, 2.15; N, 9.95. Found: C, 33.92; H, 1.94; N, 10.00. FAB-MS: $\mathrm{m} / \mathrm{z} 844\left[\mathrm{M}^{+}\right], 777\left[\mathrm{M}^{+}\right.$ - pz]. IR (Nujol): $v=1605,1563,1520 \mathrm{~cm}^{-1} .{ }^{1} \mathrm{H}$ NMR $\left(\mathrm{CD}_{2-}\right.$ $\mathrm{Cl}_{2}$ ): 6.45 (virtual t, $2 \mathrm{H}, \mathrm{H}(4)$ of $\left.\mathrm{pz}\right), 6.78-6.91(\mathrm{~m}, 4 \mathrm{H}), 7.35-$ $7.42(\mathrm{~m}, 4 \mathrm{H}), 7.62-7.78(\mathrm{~m}, 6 \mathrm{H}), 8.15$ (d with v. broad ${ }^{195} \mathrm{Pt}$ satellites, $1 \mathrm{H}, 3 \mathrm{~J} \mathrm{HH}=5.7 \mathrm{~Hz}$ ), 8.30 (d with v. broad ${ }^{195} \mathrm{Pt}$ satellites, $\left.1 \mathrm{H},{ }^{3} \mathrm{H}_{\mathrm{HH}}=6.0 \mathrm{~Hz}\right) \cdot{ }^{13} \mathrm{C}\left\{{ }^{1} \mathrm{H}\right\} \mathrm{NMR}\left(\mathrm{CDCl}_{3}\right): 105.7$, $106.0,106.1,117.5,117.6,119.1,119.4,127.6,127.8,132.8$, $133.0,138.1,138.4,138.8,138.9,139.9,140.1,149.2,149.3$, $149.4,150.1,163.6,163.9$.

[Pt(Thpy)(aza) $]_{2}$, 3a. The procedure for $\mathbf{2 a}$ was adopted using 7-azaindole $(0.04 \mathrm{~g}, 0.38 \mathrm{mmol})$, excess $\mathrm{NaH}$, and $\mathbf{1 a}$ $(0.20 \mathrm{~g}, 0.36 \mathrm{mmol})$ to afford red crystals: yield $0.13 \mathrm{~g}, 76 \%$. Anal. Calcd for $\mathrm{C}_{32} \mathrm{H}_{22} \mathrm{~N}_{6} \mathrm{~S}_{2} \mathrm{Pt}_{2}$ : C, 40.68; $\mathrm{H}, 2.35 ; \mathrm{N}, 8.89$. Found: C, 40.43; H, 2.43; N, 8.63. FAB-MS: $\mathrm{m} / \mathrm{z} 944\left[\mathrm{M}^{+}\right]$, $827\left[\mathrm{M}^{+}-\right.$aza]. IR (Nujol): $v=1607,1559 \mathrm{~cm}^{-1} .{ }^{1} \mathrm{H}$ NMR $\left(\mathrm{CD}_{2} \mathrm{Cl}_{2}\right): 6.23(\mathrm{~d}, 1 \mathrm{H}, \mathrm{J}=4.7 \mathrm{~Hz}), 6.33(\mathrm{~d}, 1 \mathrm{H}, \mathrm{J}=3.8 \mathrm{~Hz})$, $6.62(\mathrm{t}, 1 \mathrm{H}, \mathrm{J}=6.0 \mathrm{~Hz}), 6.75-7.35(\mathrm{~m}, 10 \mathrm{H}), 7.53(\mathrm{~d}, 1 \mathrm{H}, \mathrm{J}=$ $4.8 \mathrm{~Hz}), 7.60-7.90(\mathrm{~m}, 6 \mathrm{H}), 8.11$ (d with v. broad ${ }^{195} \mathrm{Pt}$ satellites, $1 \mathrm{H}, \mathrm{J}=3.0 \mathrm{~Hz}$ ), 9.71 (d with v. broad ${ }^{195} \mathrm{Pt}$ satellites, $1 \mathrm{H}, \mathrm{J}=4.8 \mathrm{~Hz}) .{ }^{13} \mathrm{C}\left\{{ }^{1} \mathrm{H}\right\} \mathrm{NMR}\left(\mathrm{DMSO}^{-d_{6}}\right)$ : 99.6-101.1, 114.2-122.2, 125.1-133.2, 135.9-142.3, 146.3-146.8, 159.2, 159.6 .

$\left[{ }^{\mathbf{n}} \mathbf{B u}_{\mathbf{4}} \mathbf{N}\right]\left[\mathbf{P t}(\mathbf{B z q n}) \mathbf{C l}_{\mathbf{2}}\right]$, 1b. The procedure for $\mathbf{1 a}$ was adopted using 7,8-benzoquinoline (0.36 g, $1.99 \mathrm{mmol}), \mathrm{K}_{2} \mathrm{PtCl}_{4}$ $(0.20 \mathrm{~g}, 0.48 \mathrm{mmol})$, and ${ }^{\mathrm{n}} \mathrm{Bu} \mathrm{u}_{4} \mathrm{NCl}(0.58 \mathrm{~g}, 2.09 \mathrm{mmol})$ to yield yellow crystals: yield $0.16 \mathrm{~g}, 50 \%$. Anal. Calcd for $\mathrm{C}_{29} \mathrm{H}_{44} \mathrm{~N}_{2-}$ $\mathrm{Cl}_{2}$ Pt: C, 50.73; $\mathrm{H}, 6.46 ; \mathrm{N}, 4.08$. Found: $\mathrm{C}, 50.96 ; \mathrm{H}, 6.45 ; \mathrm{N}$, 3.78. FAB-MS (-ve): $\mathrm{m} / \mathrm{z} 444$ [M- ]. IR (Nujol): $v=1621,1592$,

(22) Demas, J . N.; Crosby, G. A. J . Phys. Chem. 1971, 75, 991. 
$1569 \mathrm{~cm}^{-1} .{ }^{1} \mathrm{H}$ NMR $\left(\mathrm{CD}_{3} \mathrm{CN}\right): 0.91\left(\mathrm{t}, 12 \mathrm{H}, \mathrm{J}=7.3 \mathrm{~Hz}, \mathrm{CH}_{3-}\right.$ $\left.\left(\mathrm{CH}_{2}\right)_{3}\right), 1.28\left(\mathrm{~m}, 8 \mathrm{H}, \mathrm{CH}_{3}\left(\mathrm{CH}_{2}\right)_{3}\right), 1.54\left(\mathrm{~m}, 8 \mathrm{H}, \mathrm{CH}_{3}\left(\mathrm{CH}_{2}\right)_{3}\right), 3.06$ $\left(\mathrm{m}, 8 \mathrm{H}, \mathrm{CH}_{3}\left(\mathrm{CH}_{2}\right)_{3}\right), 7.43-7.83(\mathrm{~m}, 5 \mathrm{H}), 8.06$ (d with broad ${ }^{195} \mathrm{Pt}$ satellites, $\left.1 \mathrm{H}, 3_{\mathrm{HH}}=7.3 \mathrm{~Hz},{ }^{3} \mathrm{PtH}_{\mathrm{PtH}}=43 \mathrm{~Hz}\right), 8.42(\mathrm{~d}, 1 \mathrm{H}, \mathrm{J}=$ $8.0 \mathrm{~Hz}$ ), 9.99 (d with broad ${ }^{195} \mathrm{Pt}$ satellites, $1 \mathrm{H},{ }^{3} \mathrm{~J} \mathrm{HH}=5.5 \mathrm{~Hz}$, $\left.{ }^{3} \mathrm{PtH}_{\mathrm{PtH}}=47 \mathrm{~Hz}\right) .{ }^{13} \mathrm{C}\left\{{ }^{1} \mathrm{H}\right\} \mathrm{NMR}\left(\mathrm{CD}_{3} \mathrm{CN}\right): 13.6 ; 20.3 ; 24.4 ; 59.4$ $\left(\mathrm{CH}_{3}\left(\mathrm{CH}_{2}\right)_{3}\right), 120.9,122.3,124.2,127.5,129.7,129.8,130.8$, 134.3, 137.3, 140.0, 149.5, 156.6, 158.2.

[Pt(Bzqn)(pz)] $\mathbf{2}_{2} \mathbf{2 b}$. The procedure for $\mathbf{2 a}$ was adopted using pyrazole $(0.02 \mathrm{~g}, 0.29 \mathrm{mmol})$, excess $\mathrm{NaH}$, and $\mathbf{1 b}(0.18$ $\mathrm{g}, 0.26 \mathrm{mmol}$ ). A yellow solution was obtained after stirring at reflux for $12 \mathrm{~h}$ under a $\mathrm{N}_{2}$ atmosphere. Recrystallization by slow evaporation from an acetone/DMF mixture yielded yellow crystals: yield $0.08 \mathrm{~g}, 72 \%$. Anal. Calcd for $\mathrm{C}_{32} \mathrm{H}_{22} \mathrm{~N}_{6}$ $\mathrm{Pt}_{2}$ : C, 43.64; $\mathrm{H}, 2.52 ; \mathrm{N}, 9.54$. Found: $\mathrm{C}, 43.45 ; \mathrm{H}, 2.78 ; \mathrm{N}$, 9.67. FAB-MS: $\mathrm{m} / \mathrm{z} 879\left[\mathrm{M}^{+}\right], 812\left[\mathrm{M}^{+}-\mathrm{pz}\right]$. IR (Nujol): $v=$ $1621,1594,1568 \mathrm{~cm}^{-1}$. ${ }^{1} \mathrm{H}$ NMR $\left(\mathrm{CD}_{2} \mathrm{Cl}_{2}\right): 6.58$ (virtual t, $2 \mathrm{H}$, $\mathrm{H}(4)$ of pz), 7.33-7.57 (m, 10H), 7.74-7.80 (m, 4H), $7.94(\mathrm{~d}$, $2 \mathrm{H}, \mathrm{J}=7.2 \mathrm{~Hz}$ ), $8.31(\mathrm{t}, 2 \mathrm{H}, \mathrm{J}=6.6 \mathrm{~Hz}$ ), 8.67 (d with v. broad ${ }^{195} \mathrm{Pt}$ satellites, $1 \mathrm{H}, \mathrm{J}=4.9 \mathrm{~Hz}$ ), 8.79 (d with v. broad ${ }^{195} \mathrm{Pt}$ satellites, $1 \mathrm{H}, \mathrm{J}=5.3 \mathrm{~Hz}$ ). ${ }^{13} \mathrm{C}\left\{{ }^{1} \mathrm{H}\right\}$ NMR (DMSO- $\mathrm{d}_{6}$ ): 106.3, $106.4,106.5,121.2,121.3,121.9,122.0,123.2,123.4,123.5$, $126.4,128.7,128.8,129.0,129.2,129.4,130.5,132.9,138.1$, $138.3,139.1,141.4,141.5,141.8,142.6,148.1,148.3,156.0$, 156.1.

[Pt(Bzqn)(aza) $]_{2}$, 3b. The procedure for $\mathbf{2 a}$ was adopted using 7-azaindole $(0.04 \mathrm{~g}, 0.30 \mathrm{mmol})$, excess $\mathrm{NaH}$, and $\mathbf{1 b}$ $(0.18 \mathrm{~g}, 0.26 \mathrm{mmol})$. An orange solution was obtained after stirring at reflux for $12 \mathrm{~h}$ under a $\mathrm{N}_{2}$ atmosphere. Recrystallization by diffusion of diethyl ether into a dichloromethane solution yielded an orange microcrystalline solid: yield 0.09 g, 74\%. Anal. Calcd for $\mathrm{C}_{40} \mathrm{H}_{26} \mathrm{~N}_{6} \mathrm{Pt}_{2}$ : C, 48.98; $\mathrm{H}, 2.67 ; \mathrm{N}, 8.57$. Found: C, 49.13; H, 2.43; N, 8.30. FAB-MS: $\mathrm{m} / \mathrm{z} 979\left[\mathrm{M}^{+}\right]$, $862\left[\mathrm{M}^{+}-\right.$aza]. IR (Nujol): $v=1620,1596,1576 \mathrm{~cm}^{-1} .{ }^{1} \mathrm{H}$ $\operatorname{NMR}\left(\mathrm{CD}_{2} \mathrm{Cl}_{2}\right): 5.83(\mathrm{~d}, 1 \mathrm{H}, \mathrm{J}=7.4 \mathrm{~Hz}), 6.67-7.36(\mathrm{~m}, 15 \mathrm{H})$, 7.46-7.81 (m, 5H), 8.10-8.25 (m, 3H), $8.36(\mathrm{~s}, 1 \mathrm{H}), 9.12(\mathrm{~d}$ with v. broad ${ }^{195} \mathrm{Pt}$ satellites, $1 \mathrm{H}, \mathrm{J}=5.0 \mathrm{~Hz}$ ). ${ }^{13} \mathrm{C}\left\{{ }^{1} \mathrm{H}\right\} \mathrm{NMR}$ (DMSO- $\left.d_{6}\right)$ : $100.8-101.3,101.7,113.8,114.4,121.7-133.3$, 135.6-140.2, 146.8, 151.6, 156.5.

[Pt(Phpy)(pz) ] 2, 2c. The procedure for $\mathbf{2 b}$ was adopted using pyrazole $(0.02 \mathrm{~g}, 0.32 \mathrm{mmol})$, excess $\mathrm{NaH}$, and $\mathbf{1 c}(0.20$ $\mathrm{g}, 0.30 \mathrm{mmol}$ ) to afford yellow crystals: yield $0.10 \mathrm{~g}, 80 \%$. Anal. Calcd for $\mathrm{C}_{28} \mathrm{H}_{22} \mathrm{~N}_{6} \mathrm{Pt}_{2}$ : C, 40.39; $\mathrm{H}, 2.66 ; \mathrm{N}, 10.09$. Found: $\mathrm{C}$, 40.69; H, 2,42; N, 10.00. FAB-MS: $\mathrm{m} / \mathrm{z} 832\left[\mathrm{M}^{+}\right], 765\left[\mathrm{M}^{+}-\right.$ pz]. IR (Nujol): $v=1607,1584,1565 \mathrm{~cm}^{-1}$. ${ }^{1} \mathrm{H}$ NMR (DMSO$\left.\mathrm{d}_{6}\right): 6.46$ (virtual t, $2 \mathrm{H}, \mathrm{H}(4)$ of $\left.\mathrm{pz}\right), 6.89(\mathrm{~d}, 1 \mathrm{H}, \mathrm{J}=6.5 \mathrm{~Hz})$, 6.96-7.07 (m, 5H), $7.30(\mathrm{~m}, 2 \mathrm{H}), 7.58(\mathrm{~d}, 1 \mathrm{H}, \mathrm{J}=2.2 \mathrm{~Hz}), 7.69$ $(\mathrm{m}, 4 \mathrm{H}), 7.80(\mathrm{~d}, 1 \mathrm{H}, \mathrm{J}=2.0 \mathrm{~Hz}), 8.04(\mathrm{~m}, 4 \mathrm{H}), 8.27(\mathrm{~d}, 1 \mathrm{H}$, $\mathrm{J}=5.8 \mathrm{~Hz}$ ), $8.41(\mathrm{~d}, 1 \mathrm{H}, \mathrm{J}=5.7 \mathrm{~Hz}) .{ }^{13} \mathrm{C}\left\{{ }^{1} \mathrm{H}\right\} \mathrm{NMR}$ (DMSO$\left.\mathrm{d}_{6}\right)$ : $106.1,106.2,106.3,119.3,119.4,122.8,122.9,123.1,123.2$, $123.6,123.7,129.1,133.0,133.1,137.7,137.9,138.6,138.7$, $139.3,144.3,145.0,145.2,145.3,148.5,148.7,166.8,167.0$.

[Pt(Phpy)(aza) $]_{2}, 3 \mathbf{c}$. The procedure for $\mathbf{2 a}$ was adopted using 7-azaindole $(0.04 \mathrm{~g}, 0.34 \mathrm{mmol})$, excess $\mathrm{NaH}$, and $\mathbf{1 c}$ $(0.20 \mathrm{~g}, 0.30 \mathrm{mmol})$ to afford red crystals: yield $0.11 \mathrm{~g}, 79 \%$. Anal. Calcd for $\mathrm{C}_{36} \mathrm{H}_{26} \mathrm{~N}_{6} \mathrm{Pt}_{2}$ : C, 46.35; $\mathrm{H}, 2.81 ; \mathrm{N}, 9.01$. Found: C, 46.46; H, 2.79; N, 9.14. FAB-MS: m/z $932\left[\mathrm{M}^{+}\right]$, $815\left[\mathrm{M}^{+}-\right.$aza]. IR (Nujol): $v=1607,1585,1557 \mathrm{~cm}^{-1} .{ }^{1} \mathrm{H}$ NMR $\left(\mathrm{CD}_{2} \mathrm{Cl}_{2}\right): 5.93(\mathrm{~m}, 1 \mathrm{H}), 6.03(\mathrm{~d}, 1 \mathrm{H}, \mathrm{J}=7.4 \mathrm{~Hz}), 6.23$ $(\mathrm{m}, 1 \mathrm{H}), 6.44-6.81(\mathrm{~m}, 9 \mathrm{H}), 6.99(\mathrm{~d}, 2 \mathrm{H}, \mathrm{J}=7.4 \mathrm{~Hz}), 7.15-$ $7.75(\mathrm{~m}, 8 \mathrm{H}), 7.91-7.99(\mathrm{~m}, 2 \mathrm{H}), 8.47$ (d with v. broad ${ }^{195} \mathrm{Pt}$ satellites, $2 \mathrm{H}, \mathrm{J}=5.1 \mathrm{~Hz}$ ). ${ }^{13} \mathrm{C}\left\{{ }^{1} \mathrm{H}\right\}$ NMR (DMSO-d 6 ): 100.1, 100.2 , 101.3, 113.4, 117.7, 121.3-124.4, 128.1-131.0, 138.1$140.4,142.5,144.7,149.3,159.9$.

X-ray crystallography. Crystals of $\mathbf{1 a}$ and $\mathbf{2 a}$ were grown by vapor diffusion of diethyl ether into dichloromethane solutions. Crystal data and details of collection and refinement are summarized in Table 1.

For $\mathbf{l a}$, diffraction experiments were performed on a Rigaku AFC7R diffractometer ( $\lambda=0.71073 \AA, \omega-2 \theta$ scans). The structure was solved by Patterson methods, expanded by
Table 1. Crystal Data

\begin{tabular}{|c|c|c|}
\hline & 1a & $2 a$ \\
\hline $\begin{array}{l}\text { formula } \\
\text { fw }\end{array}$ & $\begin{array}{l}\mathrm{C}_{18} \mathrm{H}_{13} \mathrm{~N}_{2} \mathrm{~S}_{2} \mathrm{PtCl} \\
551.98\end{array}$ & $\begin{array}{l}\mathrm{C}_{24} \mathrm{H}_{18} \mathrm{~N}_{6} \mathrm{~S}_{2} \mathrm{Pt}_{2} \\
844.75\end{array}$ \\
\hline color & orange & orange \\
\hline crystal size, $\mathrm{mm}$ & $0.30 \times 0.20 \times 0.08$ & $0.45 \times 0.25 \times 0.15$ \\
\hline crystal system & monodinic & monoclinic \\
\hline space group & $\mathrm{P} 2_{1} / \mathrm{n}$ (No. 14) & $\mathrm{P} 22_{1} / \mathrm{C}$ \\
\hline$a, \AA$ & $9.078(2)$ & $17.215(2)$ \\
\hline $\mathrm{b}, \AA$ & $8.363(1)$ & $9.204(1)$ \\
\hline$c, \AA$ & $23.562(7)$ & $16.735(2)$ \\
\hline$\beta$, deg & $94.57(2)$ & $116.466(8)$ \\
\hline $\mathrm{V}, \AA^{3}$ & 1783.3(6) & $2373.8(4)$ \\
\hline $\mathrm{Z}$ & 4 & 4 \\
\hline$D_{c}\left(g ~ c m^{-3}\right)$ & 2.056 & 2.364 \\
\hline$\mu, \mathrm{cm}^{-1}$ & 82.22 & 120.93 \\
\hline$F(000)$ & 1048 & 1567 \\
\hline $\mathrm{T}, \mathrm{K}$ & 301 & 295 \\
\hline $2 \theta_{\max }$, deg & 50 & 50 \\
\hline no. of unique data & 3381 & 4177 \\
\hline no. of obsd data & $2666[1>3 \sigma(I)]$ & $2530[1>2 \sigma(I)]$ \\
\hline no. of variables & 217 & 361 \\
\hline $\mathrm{R}, \mathrm{R}_{\mathrm{w}}^{\mathrm{b}}$ & $0.037,0.045$ & $0.031,0.027$ \\
\hline residual & $+1.30,-0.94$ & $+1.20,-0.62$ \\
\hline
\end{tabular}

Fourier methods (PATTY ${ }^{23}$ ), and refined by full-matrix leastsquares using the software package TeXsan ${ }^{24}$ on a Silicon Graphics Indy computer. One formula unit constitutes a crystallographic asymmetric unit. All 24 non-H atoms were refined anisotropically, and $13 \mathrm{H}$ atoms at calculated positions were not refined.

For $\mathbf{2 a}$, diffraction experiments were performed on a Nonius diffractometer ( $\lambda=0.71073 \AA, \theta / 2 \theta$ scans). The structure was solved by direct methods and refined by least-squares treatment on $\mathrm{F}^{2}$ using the NRCVAX program. One formula unit constitutes a crystallographic asymmetric unit. All 40 non-H atoms were refined anisotropically, and $26 \mathrm{H}$ atoms at calculated positions were not refined. The two 2-(2'-thienyl)pyridyl groups are disordered with $\mathrm{NC}(1), \mathrm{NC}(2), \mathrm{NC}(3)$, and $\mathrm{NC}(4)$ having half carbon and nitrogen atom occupancy and $S(1), S\left(1^{\prime}\right), S(2), S\left(2^{\prime}\right), C(6), C\left(6^{\prime}\right), C(7), C\left(7^{\prime}\right), C(20), C\left(20^{\prime}\right)$, $\mathrm{C}(21)$, and $\mathrm{C}\left(21^{\prime}\right)$ also having half occupancy.

\section{Results and Discussion}

Synthesis and Characterization. A general synthetic route to bi- and triplatinum macrocycles is illustrated in Scheme 1. The monomeric precursors la-c (a, Thpy; b, Bzqn; c, Phpy) comprise two substitutionally labile ligands (HThpy and/or chloride). Treatment with stoichiometric amounts of deprotonated $\mathrm{N}$-donor ligands in $\mathrm{CH}_{2} \mathrm{Cl}_{2} / \mathrm{THF}$ under a nitrogen atmosphere afford cyclic dimers with $\mu$-pz (denoted 2) and $\mu$-aza (denoted 3) linkers, while trimers are formed with $\mu$-bzim groups (denoted 4). Syntheses of compounds based on the $\left[\mathrm{Pt}(\mu-\mathrm{pz})_{2} \mathrm{Pt}\right]$ core have been reported. ${ }^{2 \mathrm{a}-\mathrm{c}}$ All complexes are air- and moisturestable at room temperature in solid and solution states.

These reactions to form macrocycles proceed in moderately high yields (70-90\%). We have al ready established $^{17}$ that the coordination geometry of the bzim ligand is suitable for the assembly of trimeric metallo-

(23) PATTY: Beurskens, P. R.; Admiraal, G.; Bosman, W. P.; GarciaGranda, S.; Gould, R. O.; Smits, J. M. M.; Smykalla, C. The DIRDIF program system; Technical Report of the Crystallography Laboratory, University of Nijmegen: The Netherlands,1992.

(24) TeXsan: Crystal Structure Analysis Package; Molecular Structure Corporation: The Woodlands, TX, 1985 and 1992. 
Scheme 1. Synthetic Route toward Bi-and Trimetallic Macrocycles

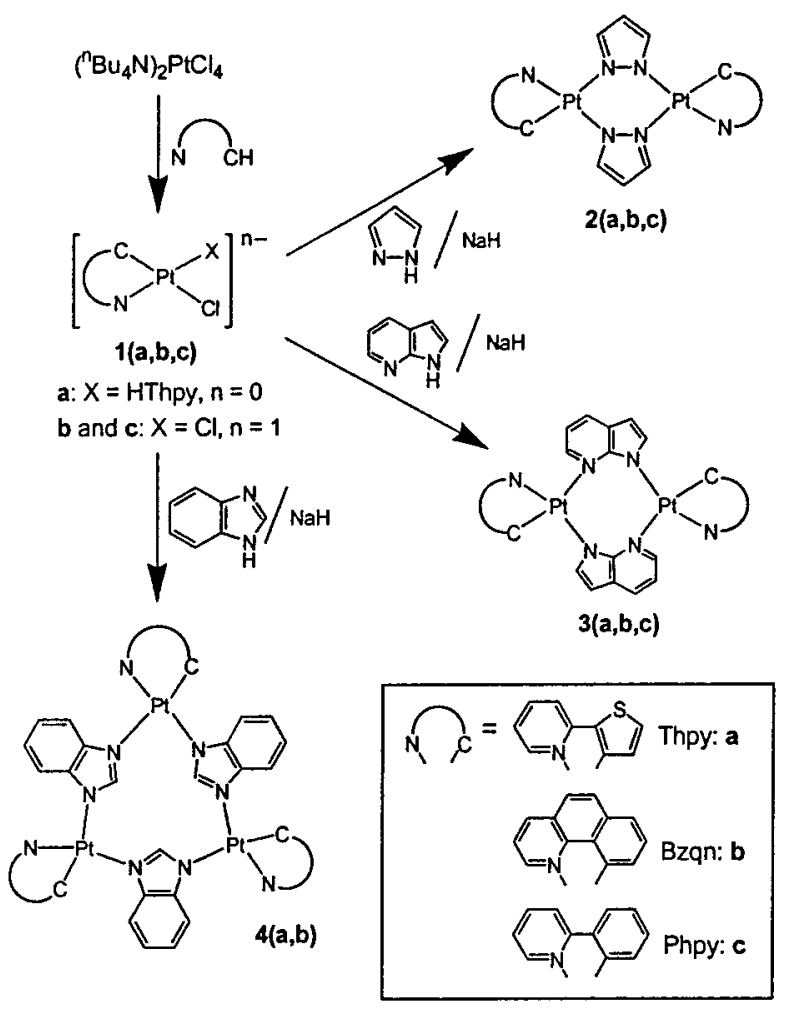

Scheme 2. Ligand-Directed Strategy toward Trimetallic Macrocycles<smiles>C[P+]1(C)CCCN1</smiles><smiles></smiles>

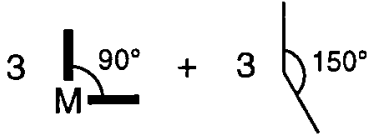

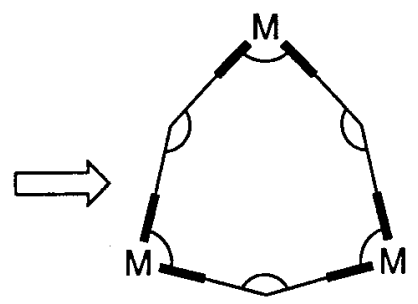

macrocycles, by coupling of three $150^{\circ}$ edges (as for bzim) and three cis binding sites at square planar and octahedral metal centers (Scheme 2). Our unsuccessful attempts to synthesize $[\mathrm{Pt}(\mathrm{Phpy})(\mathrm{bzim})]_{3}$, which afforded insoluble, intractable solids, are therefore surprising. Nevertheless, the intrinsic binding geometry of the bidentate $\mathrm{pz}$ and aza ligands evidently ensure the efficient and selective formation of dimeric Pt(II) complexes. The positive FAB mass spectra of the cyclic dimers and trimers reveal clusters corresponding to the molecular ion, while no other oligomeric species are detected. The ${ }^{1} \mathrm{H}$ NMR spectra for all complexes contain distinctive low-field doublets with broad ${ }^{195} \mathrm{Pt}$ satellites (3) PtH $\sim 40 \mathrm{~Hz}$ ) which are assigned to $\mathrm{H}_{\beta}$ of the cyclometalating ligands. Variable-temperature ${ }^{1} \mathrm{H}$ NMR studies ( -60 to $40{ }^{\circ} \mathrm{C}$ in $\mathrm{CD}_{2} \mathrm{Cl}_{2}$ ) were performed on $\mathbf{4 a}$ to investigate the orientation of the bzim ligands in solution. No significant changes were detected, but the syn, anti, anti conformation in the solid state (see below) is unlikely to be maintained in solution.

Crystal Structures. In this work, the molecular structures of the mononuclear complex 1a and $\mu$-pyrazolate dimer $\mathbf{2 a}$ (Figures 1 and 2, respectively) were
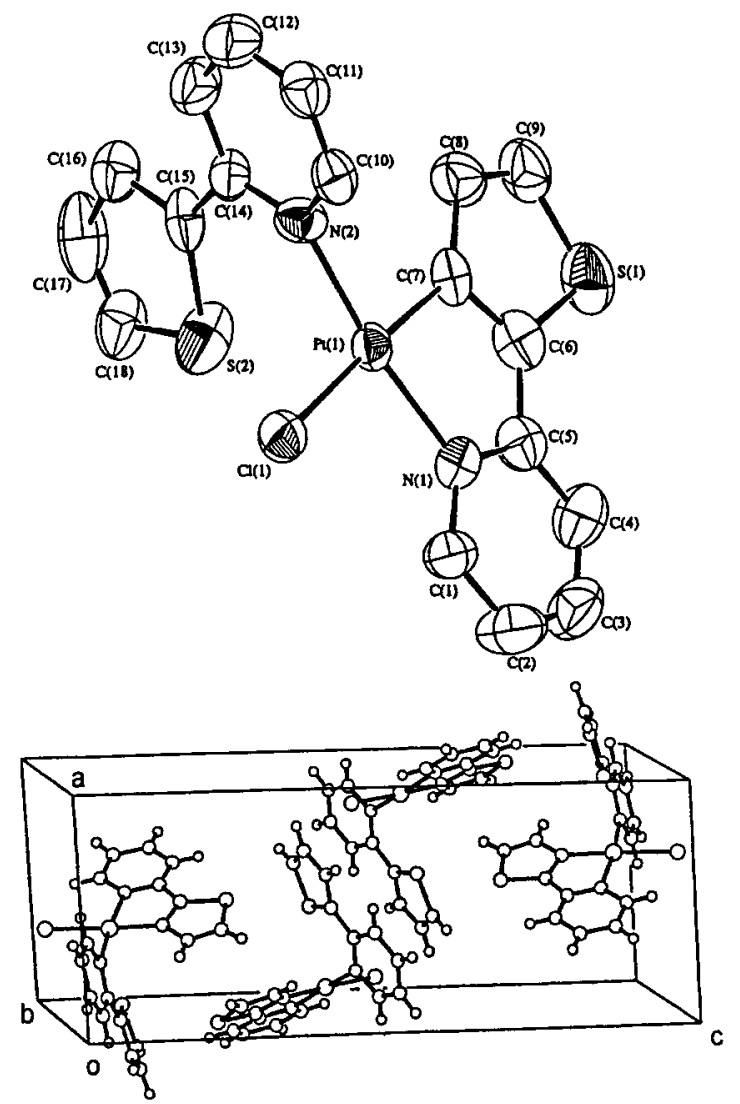

Figure 1. (Top) Perspective view of [Pt(Thpy)(HThpy)$\mathrm{Cl}$, 1a, with $50 \%$ probability ellipsoids. (Bottom) Crystal packing diagram showing $\pi-\pi$ stacking interactions.

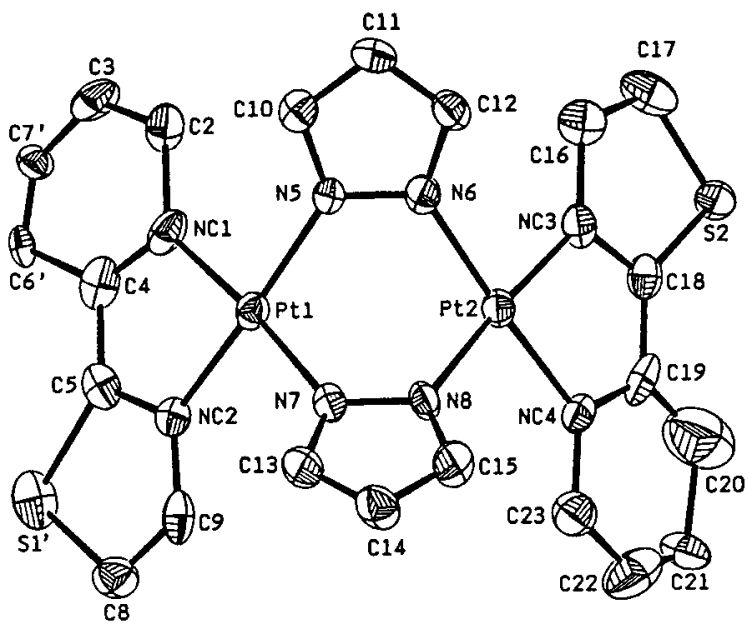

Figure 2. Perspective view of $[\mathrm{Pt}(\mathrm{Thpy})(\mathrm{pz})]_{2}$, 2a, with $35 \%$ probability ellipsoids. Thpy ligands ( $\mathrm{NC}(1-4), \mathrm{S}\left(1^{\prime}\right)$, $\mathrm{S}(2), \mathrm{C}\left(6^{\prime}\right), \mathrm{C}\left(7^{\prime}\right), \mathrm{C}(20), \mathrm{C}(21)$ atoms) are disordered.

determined by X-ray crystallography (Table 2). We shall also elaborate on the solid-state structure of $\mathbf{4 a}$.

The platinum atom in $\mathbf{1 a}$ resides in an approximately square planar environment and is coordinated to monodentate (pyridyl $\mathrm{N}$ ) and bidentate (pyridyl $\mathrm{N}$ and thienyl C) 2-(2'-thienyl)pyridyl ligands plus a chloride group (Figure 1). The Pt(1)-Cl(1) bond length of 2.402(2) $\AA$ is partially longer than that reported for $\mathrm{Pt}(\mathrm{bpy}) \mathrm{Cl}_{2}$ $(2.306(2) \AA)^{25}$ due to the greater trans influence of the

(25) Osborn, R. S.; Rogers, D. J . Chem. Soc., Dalton Trans. 1974, $(25)$ 
Table 2. Selected Bond Lengths $(\AA \AA)$ and Angles (deg)

\begin{tabular}{lccc}
\hline \multicolumn{5}{c}{ Complex 1a } \\
$\mathrm{Pt}(1)-\mathrm{Cl}(1)$ & $2.402(2)$ & $\mathrm{Pt}(1)-\mathrm{C}(7)$ & $1.990(9)$ \\
$\mathrm{Pt}(1)-\mathrm{N}(1)$ & $2.060(7)$ & $\mathrm{S}(1)-\mathrm{C}(6)$ & $1.73(1)$ \\
$\mathrm{Pt}(1)-\mathrm{N}(2)$ & $2.032(7)$ & $\mathrm{S}(1)-\mathrm{C}(9)$ & $1.71(1)$ \\
$\mathrm{N}(1)-\mathrm{Pt}(1)-\mathrm{C}(7)$ & $80.4(3)$ & $\mathrm{Cl}(1)-\mathrm{Pt}(1)-\mathrm{N}(2)$ & $89.3(2)$ \\
$\mathrm{Cl}(1)-\mathrm{Pt}(1)-\mathrm{N}(1)$ & $97.3(2)$ & $\mathrm{Cl}(1)-\mathrm{Pt}(1)-\mathrm{C}(7)$ & $177.3(3)$ \\
\multicolumn{4}{c}{} \\
$\mathrm{Pt}(1)-\mathrm{NC}(1)$ & $\mathrm{C}$ Complex 2a \\
$\mathrm{Pt}(1)-\mathrm{NC}(2)$ & $1.995(9)$ & $\mathrm{Pt}(2)-\mathrm{N}(6)$ & $2.031(8)$ \\
$\mathrm{Pt}(1)-\mathrm{N}(5)$ & $2.986(9)$ & $\mathrm{Pt}(2)-\mathrm{N}(8)$ & $2.043(8)$ \\
$\mathrm{Pt}(1)-\mathrm{N}(7)$ & $2.032(8)$ & $\mathrm{S}(2)-\mathrm{C}(17)$ & $1.799(15)$ \\
$\mathrm{Pt}(2)-\mathrm{NC}(3)$ & $2.056(8)$ & $\mathrm{S}(2)-\mathrm{C}(18)$ & $1.72(1)$ \\
$\mathrm{Pt}(2)-\mathrm{NC}(4)$ & $2.017(8)$ & $\mathrm{S}\left(1^{\prime}\right)-\mathrm{C}(5)$ & $1.88(1)$ \\
$\mathrm{NC}(1)-\mathrm{Pt}(1)-\mathrm{NC}(2)$ & $79.1(4)$ & $\mathrm{SC}\left(1^{\prime}\right)-\mathrm{C}(8)$ & $1.804(14)-\mathrm{Pt}(2)-\mathrm{N}(8)$ \\
$\mathrm{NC}(1)-\mathrm{Pt}(1)-\mathrm{N}(5)$ & $96.6(4)$ & $\mathrm{N}(6)-\mathrm{Pt}(2)-\mathrm{N}(8)$ & $96.3(3)$ \\
$\mathrm{NC}(2)-\mathrm{Pt}(1)-\mathrm{N}(7)$ & $95.9(3)$ & $\mathrm{Pt}(1)-\mathrm{N}(5)-\mathrm{N}(6)$ & $128.3(3)$ \\
$\mathrm{N}(5)-\mathrm{Pt}(1)-\mathrm{N}(7)$ & $88.4(3)$ & $\mathrm{Pt}(2)-\mathrm{N}(6)-\mathrm{N}(5)$ & $120.8(6)$ \\
$\mathrm{NC}(3)-\mathrm{Pt}(2)-\mathrm{NC}(4)$ & $79.5(4)$ & $\mathrm{C}(5)-\mathrm{S}\left(1^{\prime}\right)-\mathrm{C}(8)$ & $90.9(6)$ \\
$\mathrm{NC}(3)-\mathrm{Pt}(2)-\mathrm{N}(6)$ & $95.9(4)$ & $\mathrm{C}(17)-\mathrm{S}(2)-\mathrm{C}(18)$ & $92.5(6)$
\end{tabular}

thienyl carbanion compared to the pyridyl $\mathrm{N}$ donor. The stacked arrangement of 1a monomers (bottom of Figure 1) indicates $\pi-\pi$ interactions between adjacent monodentate HThpy ligands, with interplanar separations of ca. 3.5 $\AA$. The structure of the iodi de congener has been reported. ${ }^{26}$

In $\mathbf{2 a}$, two [Pt(Thpy)] moieties are bridged by two pyrazolate ligands in an exo-bidentate fashion (Figure 2). The central six-membered [Pt $\left.(\mu-\mathrm{pz})_{2} \mathrm{Pt}\right]$ core is nonplanar, with mean $\mathrm{Pt}-\mathrm{N}(\mathrm{pz})$ distances of $2.04 \AA$ and $\mathrm{N}-\mathrm{Pt}-\mathrm{N}$ angles of $\mathrm{ca}$. $88^{\circ}$ between the pz groups. The metal atoms are positioned at the vertexes of a boat conformation like in previously reported $\mathrm{Pt}(\mathrm{II})^{2 \mathrm{~b}, \mathrm{c}}$ and $\operatorname{Ir}(\mathrm{I})^{27} \mu$-pyrazol ate dimers. The Pt(1)-Pt(2) separation of 3.4863(6) $\AA$ in $\mathbf{2 a}$ is comparable to that in the mono(pyrazolyl) species $\left[\mathrm{Pt}_{2}(\mathrm{tpy})_{2}(\mu-\mathrm{pz})\right]\left(\mathrm{ClO}_{4}\right)_{3}(3.432(1) \AA),{ }^{28}$ but is longer than the corresponding metal-metal distance in $\left[\mathrm{PtCl}\left(\mathrm{PPh}_{2} \mathrm{Me}\right)\left(u-3,5-\mathrm{Me} \mathrm{e}_{2} \mathrm{pz}\right)\right]_{2}(3.170(1) \AA){ }^{2 \mathrm{C}}$ Comparable bond lengths within the pyrazolate rings suggest substantial delocalization upon deprotonation. The bite angles of the cyclometalating Thpy ligands $\left(79.1(4)^{\circ}\right.$ and $\left.79.5(4)^{\circ}\right)$ are comparable to that in $\mathbf{l a}$ $\left(80.4(3)^{\circ}\right)$.

The "two-up, one-down" orientation of the bzim ligands in the mol ecular structure of $\mathbf{4 a}$, which allows comparisons with the partial cone conformation in calixarenes ${ }^{29}$ and the $\mathrm{Pt}(\mathrm{II})$-incorporated analogue of calix[4]arene $[\mathrm{Pt}(\mathrm{en}) \text { (uracilate) }]_{4}{ }^{4+}$ (en $=1,2$-diaminoethane), ${ }^{30}$ has been described previously. ${ }^{17}$ From the partial crystal packing diagram (Figure 3 ), $\pi-\pi$ interactions of ca. 3.5 $\AA$ between Thpy systems in adjacent molecules are apparent, and these are expected to influence the photophysical behavior of $\mathbf{4 a}$ in the solid state (see below).

Absorption and Emission Spectroscopy. The UV-visible spectral data of the Pt(II) complexes are listed in Table 3. Their emission data in fluid solution

(26) Giordano, T.J .; Rasmussen, P. G. Inorg. Chem. 1975, 14, 1628. (27) Coleman, A. W.; Eadie, D. T.; Stodart, S. R.; Zaworotko, M. J .; Atwood, J. L. J. Am. Chem. Soc. 1982, 104, 922.

(28) Bailey, J . A.; Gray, H. B. Acta Crystallogr. 1992, C48, 1420.

(29) Gutsche, C. D. Calixarenes; Royal Society of Chemistry: Cambridge, 1989

(30) Rauter, H.; Hillgeris, E. C.; Erxleben, A.; Lippert, B. J . Am. Chem. Soc. 1994, 116, 616

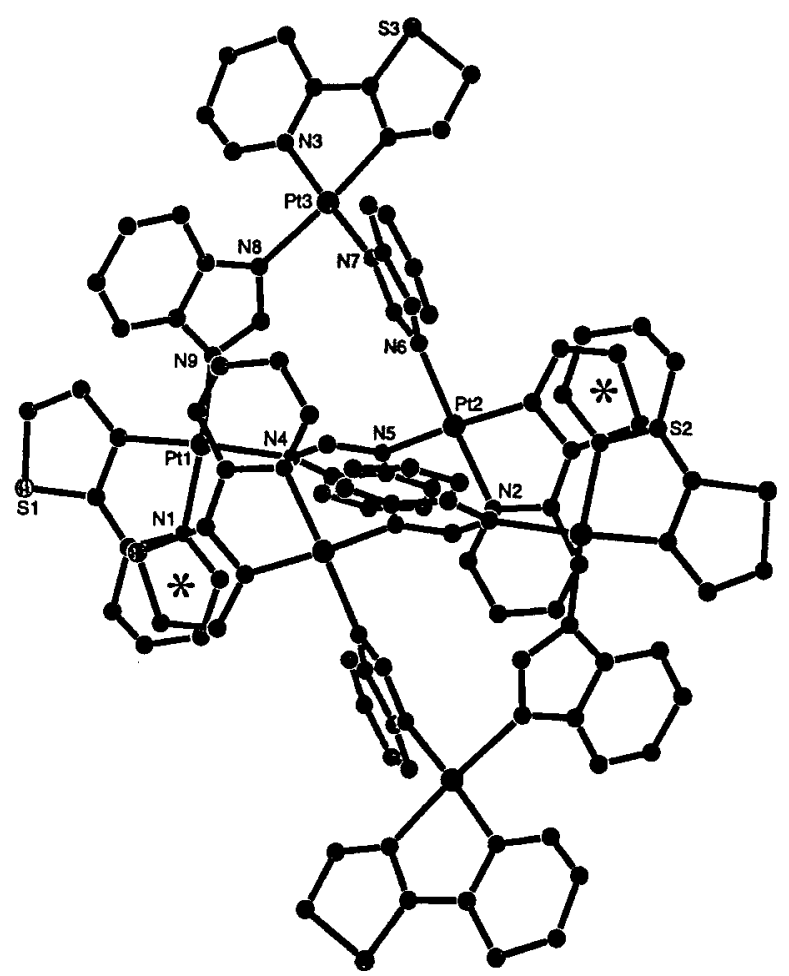

Figure 3. Partial packing diagram of $\left[\mathrm{Pt}(\text { Thpy)(bzim) }]_{3}\right.$ $4 \mathbf{a}(\pi-\pi$ interactions indicated by asterisks).

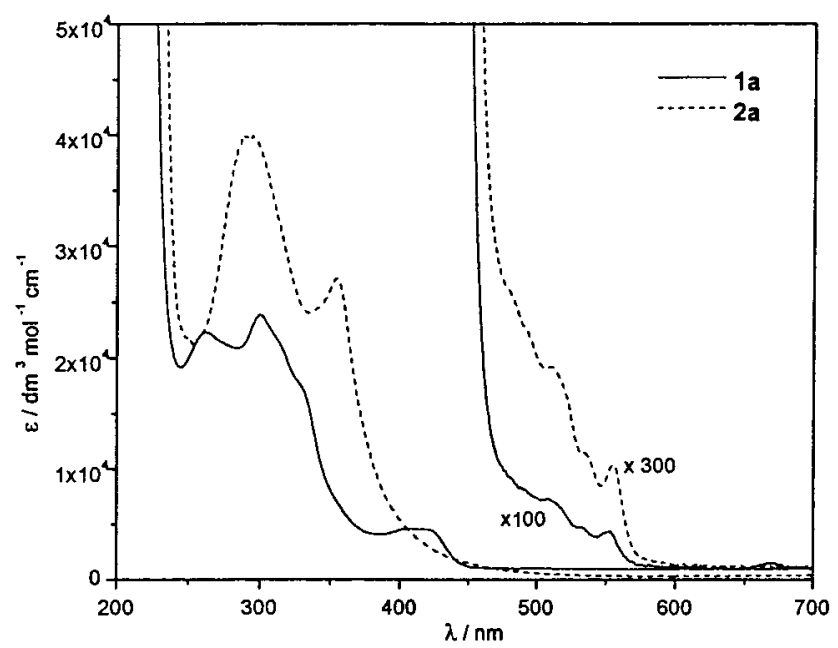

Figure 4. UV-vis absorption spectra of $\mathbf{1 a}$ and $\mathbf{2 a}$ in dichloromethane at $298 \mathrm{~K}$.

and in crystalline state are given in Tables 4 and 5, respectively.

Complexes with Thpy Ligands. The UV-vis absorption spectra of $\mathbf{2}-\mathbf{4 a}$ in $\mathrm{CH}_{2} \mathrm{Cl}_{2}$ are comparable to that for the precursor $\mathbf{1 a}$ (Figure 4 for $\mathbf{1 a}$ and $\mathbf{2 a}$ ). F or example, the spectrum of $\mathbf{4 a}$ contains high-energy bands $(\lambda<360 \mathrm{~nm})$ which are dominated by spin-allowed intraligand ( ${ }^{1} \mathrm{~L}$ : $\pi$ (Thpy) $\rightarrow \pi^{*}$ (Thpy) with metal perturbation) transitions. ${ }^{31}$ The moderately intense lowenergy bands with $\lambda_{\text {max }}$ in the range $409-421 \mathrm{~nm}$ and the notably weaker, structured absorptions at $\lambda_{\max } 480$ $554 \mathrm{~nm}\left(\epsilon<120 \mathrm{dm}^{3} \mathrm{~mol}^{-1} \mathrm{~cm}^{-1}\right)$ are assigned to the spin-allowed and spin-forbidden metal-to-ligand charge transfer ( ${ }^{1}$ MLCT and ${ }^{3}$ MLCT: (5d)Pt $\rightarrow \pi^{*}$ (Thpy)) transitions, respectively.

(31) Kvam, P.-I.; Puzyk, M. V.; Cotlyr, V. S.; Balashev, K. P.; Songstad, J . Acta Chem. Scand. 1995, 49, 645. 
Table 3. UV-Vis Absorption Data in Dichloromethane at $298 \mathrm{~K}$

\begin{tabular}{|c|c|}
\hline complex & $\lambda_{\max } / \mathrm{nm}\left(\epsilon / \mathrm{dm}^{3} \mathrm{~mol}^{-1} \mathrm{~cm}^{-1}\right)$ \\
\hline [Pt(Thpy)(HThpy)Cl], la & 262 (22300), 300 (23900), 329 (sh, 17600), 406 (4600), 419 (4550), 509 (60), $553(40)$ \\
\hline$[\mathrm{Pt}(\mathrm{Thpy})(\mathrm{pz})]_{2}, \mathbf{2 a}$ & 293 (39400), 354 (26800), 410 (sh, 3800), $512(60), 555(40)$ \\
\hline$[\mathrm{Pt}(\text { Thpy })(\mathrm{aza})]_{2}, \mathbf{3 a}$ & 292 (42900), 354 (22800), 474 (sh, 2100) \\
\hline$[\mathrm{Pt}(\mathrm{Thpy})(\mathrm{bzim})]_{3}, \mathbf{4 a}$ & 277 (47000), 337 (22000), 357 (18300), 409 (5250), 421 (4800), 480 (110), 512 (100), 554 (70) \\
\hline$\left[{ }^{\mathrm{B}} \mathrm{Bu}_{4} \mathrm{~N}\right]\left[\mathrm{Pt}(\mathrm{Bzqn}) \mathrm{Cl}_{2}\right], \mathbf{1 b}$ & 280 (28700), 358 (4350), 374 (4400), 434 (1300) \\
\hline$[\mathrm{Pt}(\mathrm{Bzqn})(\mathrm{pz})]_{2}, \mathbf{2} \mathbf{b}$ & 286 (35100), 370 (12300), 388 (sh, 8850), 421 (sh, 3150), 465 (sh, 250) \\
\hline$[\mathrm{Pt}(\mathrm{Bzqn})(\mathrm{aza})]_{2}, \mathbf{3 b}$ & 285 (34400), 380 (sh, 8700$), 410$ (sh, 4000) \\
\hline$[\mathrm{Pt}(\text { Bzqn })(\mathrm{bzim})]_{3}, \mathbf{4 b}$ & 271 (70000), 278 (73000), 358 (14000), 372 (14000), 413 (4000), 469 (sh, 440) \\
\hline$\left[{ }^{n} \mathrm{Bu}_{4} \mathrm{~N}\right]\left[\mathrm{Pt}(\mathrm{Phpy}) \mathrm{Cl}_{2}\right], \mathbf{1 c}$ & 259 (23700), 287 (15200), 331 (6300), 379 (4200), 426 (sh, 780), 490 (120) \\
\hline$[\mathrm{Pt}(\mathrm{Phpy})(\mathrm{pz})]_{2}, \mathbf{2 c}$ & 257 (45600), 284 (24500), 329 (12200), 359 (11000), 406 (3350), 446 (sh, 310) \\
\hline$[\mathrm{Pt}(\mathrm{Phpy})(\mathrm{aza})]_{2}, \mathbf{3 c}$ & 266 (33600), 290 (36200), 345 (sh, 17500), 410 (sh, 3450), 459 (sh, 1400) \\
\hline
\end{tabular}

Table 4. Emission Data in $\mathrm{CH}_{2} \mathrm{Cl}_{2}$ (complex concentration $5 \times 10^{-5} \mathrm{M}$ )

\begin{tabular}{|c|c|c|}
\hline complex & $298 \mathrm{~K}: \lambda_{\max } / \mathrm{nm} ; \tau_{o} / \mu \mathrm{s} ; \phi_{o}$ & $77 \mathrm{~K}: \lambda_{\max } / \mathrm{nm}$ \\
\hline [Pt(Thpy)(HThpy)Cl], $\mathbf{1 a}$ & 556 (max), 577 (sh), 602, 628 (sh), 661 (sh); 12.0; 0.19 & 553 (max), 573 (sh), 600, 624 (sh), 654 (sh) \\
\hline$[\mathrm{Pt}(\mathrm{Thpy})(\mathrm{pz})]_{2}, \mathbf{2} \mathbf{a}$ & 559 (max), 581 (sh), 606, 632 (sh), 664 (sh); 15.5; 0.18 & 557 (max), 576 (sh), 602, 624 (sh), 655 (sh) \\
\hline$[\mathrm{Pt}(\text { Thpy })(\mathrm{aza})]_{2}, \mathbf{3 a}$ & 561 (max), 579 (sh), 606, 638 (sh), 661 (sh); 3.1; 0.012 & 557 (max), 578 (sh), 602, 662 (sh) \\
\hline$[\mathrm{Pt}(\text { Thpy })(\mathrm{bzim})]_{3}, \mathbf{4 a}$ & 560 (max), 580 (sh), 606, 636 (sh), 661 (sh); 10.9; 0.016 & 557 (max), 575 (sh), 601, 633 (sh), 656 (sh) \\
\hline$\left[{ }^{\mathrm{B}} \mathrm{Bu}_{4} \mathrm{~N}\right]\left[\mathrm{Pt}(\mathrm{Bzqn}) \mathrm{Cl}_{2}\right], \mathbf{1 b}$ & nonemissive & $494,532,572,620$ (sh) \\
\hline$[\operatorname{Pt}(\operatorname{Bzqn})(\mathrm{pz})]_{2}, \mathbf{2} \mathbf{b}$ & $497,535,588$ (sh); $1.6 ; 9.9 \times 10^{-3}$ & $486,522,560$ \\
\hline$[\mathrm{Pt}(\text { Bzqn })(\mathrm{aza})]_{2}, \mathbf{3 b}$ & nonemissive & $497,530,565$ \\
\hline$[\mathrm{Pt}(\text { Bzqn })(\mathrm{bzim})]_{3}, \mathbf{4 b}$ & nonemissive & nonemissive \\
\hline$\left[{ }^{n} \mathrm{Bu}_{4} \mathrm{~N}\right]\left[\mathrm{Pt}(\mathrm{Phpy}) \mathrm{Cl}_{2}\right], \mathbf{1 c}$ & nonemissive & 485 (max), 527, 557, 578, 604, 631 (sh), 668 (sh) \\
\hline$[P t(P h p y)(p z)]_{2}, \mathbf{2 c}$ & $487,522,556,600$ (sh); 1.3; 0.039 & 485 (max), 515, 544, 596 (sh) \\
\hline$[\mathrm{Pt}(\text { Phpy })(\text { aza })]_{2}, 3 \mathrm{c}$ & $494,520,556,601(\mathrm{sh}) ; 0.29 ; 4.5 \times 10^{-4}$ & 489, 523 (max), 557, 605 (sh) \\
\hline
\end{tabular}

Table 5. Solid-State E mission Data

\begin{tabular}{|c|c|c|}
\hline complex & $298 \mathrm{~K}: \lambda_{\max } / \mathrm{nm}$ & $77 \mathrm{~K}: \lambda_{\max } / \mathrm{nm}$ \\
\hline [Pt(Thpy)(HThpy)Cl], $\mathbf{l a}$ & $563,581,606$ (max), 632, 651 & 574 (max), 598, 625, 652 (sh), 690 (sh) \\
\hline$[\mathrm{Pt}(\mathrm{Thpy})(\mathrm{pz})]_{2}, \mathbf{2 a}$ & 570 (sh), 591 (sh), 616 (max), 665 & 577 (max), 595, 624, 651 (sh) \\
\hline$[\mathrm{Pt}(\text { Thpy })(\text { aza })]_{2}, \mathbf{3 a}$ & 556,601 (max), 658 & 552 (max), 599, 677 \\
\hline$[\mathrm{Pt}(\mathrm{Thpy})(\mathrm{bzim})]_{3}, \mathbf{4 a}$ & $571,617,664$ (max) & 579 (max), 603 (sh), 630, 659 (sh), 691 (sh) \\
\hline$\left[{ }^{n} \mathrm{Bu}_{4} \mathrm{~N}\right]\left[\mathrm{Pt}(\mathrm{Bzqn}) \mathrm{Cl}_{2}\right], \mathbf{1 b}$ & 525 (sh), 542 (max), 591 (sh) & 514 (max), 554, 597 (sh) \\
\hline$[\mathrm{Pt}(\mathrm{Bzqn})(\mathrm{pz})]_{2}, \mathbf{2} \mathbf{b}$ & 533,568 (max), 633 & 534 (max), 568, 615 (sh) \\
\hline$[\mathrm{Pt}(\mathrm{Bzqn})(\mathrm{aza})]_{2}, \mathbf{3 b}$ & 550 (max), 592, 640 (sh) & 517,548 (max), 598, 632 (sh) \\
\hline$[\mathrm{Pt}(\text { Bzqn })(\mathrm{bzim})]_{3}, \mathbf{4 b}$ & nonemissive & 512 (max), 549, 593 (sh) \\
\hline$\left[{ }^{n} \mathrm{Bu}_{4} \mathrm{~N}\right]\left[\mathrm{Pt}(\mathrm{Phpy}) \mathrm{Cl}_{2}\right], \mathbf{1 c}$ & $502,536,566$ (sh) & 498 (max), 515, 526, 536, 558, 569 \\
\hline$[\mathrm{Pt}(\mathrm{Phpy})(\mathrm{pz})]_{2}, \mathbf{2 c}$ & $505,539,557$ (sh) & $506,526,549$ (max), 596 (sh) \\
\hline$[\mathrm{Pt}(\text { Phpy })(\text { aza })]_{2}, \mathbf{3 c}$ & $490,524,555$ & $493,528,563$ \\
\hline
\end{tabular}

Structured emissions are observed for complexes 1-4a in $\mathrm{CH}_{2} \mathrm{Cl}_{2}$ at room temperature, with peak maxima in the range 556-561 (sh 577-581) and 602606 (sh 628-638, 661-664) nm (Table 4). Minor solvatochromic effects are detected for the emission energy, e.g., for $4 a, \lambda_{\max }=564\left(\mathrm{C}_{6} \mathrm{H}_{6}\right), 560\left(\mathrm{CH}_{2} \mathrm{Cl}_{2}\right), 562\left(\mathrm{CH}_{3}-\right.$ $\mathrm{CN}), 567 \mathrm{~nm}\left(\mathrm{CH}_{3} \mathrm{OH}\right)$. Self-quenching of the emission has been detected for 1-4a; in each case, a linear plot of $1 / \tau$ against complex concentration was obtained. For example, $\mathrm{k}_{\mathrm{q}}$ values of $3.5 \times 10^{7}$ and $3.0 \times 10^{7} \mathrm{M}^{-1} \mathrm{~s}^{-1}$ have been obtained for $\mathbf{2 a}$ and $\mathbf{4 a}$, respectively. The $\mu-\mathrm{pz}$ complex $\mathbf{2 a}$ emits with a longer intrinsic lifetime $\left(\tau_{\mathrm{o}}=\right.$ $15.5 \mu \mathrm{s})$ and a significantly higher quantum yield (0.18) than the $\mu$-aza dimer $3 \mathrm{a}\left(\tau_{\mathrm{o}}=3.1 \mu \mathrm{s}, \phi_{\mathrm{o}}=0.012\right)$ and the trimer $4 \mathbf{a}\left(\tau_{\mathrm{o}}=10.9 \mu \mathrm{s}, \phi_{0}=0.016\right)$. Indeed, the photoluminescent properties displayed by $\mathbf{2 a}$ are substantially superior to those for previously reported binuclear Pt(II) luminophores, which are weakly emissive in solution at room temperature. ${ }^{19}$ At $77 \mathrm{~K}$, emission bands are slightly blue-shifted to $\lambda_{\max } 553-557$ (sh 573-578) and 600-602 (sh 624-662) nm. With reference to earlier work ${ }^{31,32}$ and the absence of any major solvatochromic effects $(<10 \mathrm{~nm})$, we suggest that these emissions are more appropriately assigned to transitions with mixed MLCT and IL character.

(32) Maestri, M.; Sandrini, D.; Balzani, V.; Chassot, L.; J olliet, P.; von Zelewsky, A. Chem. Phys. Lett. 1985, 122, 375.

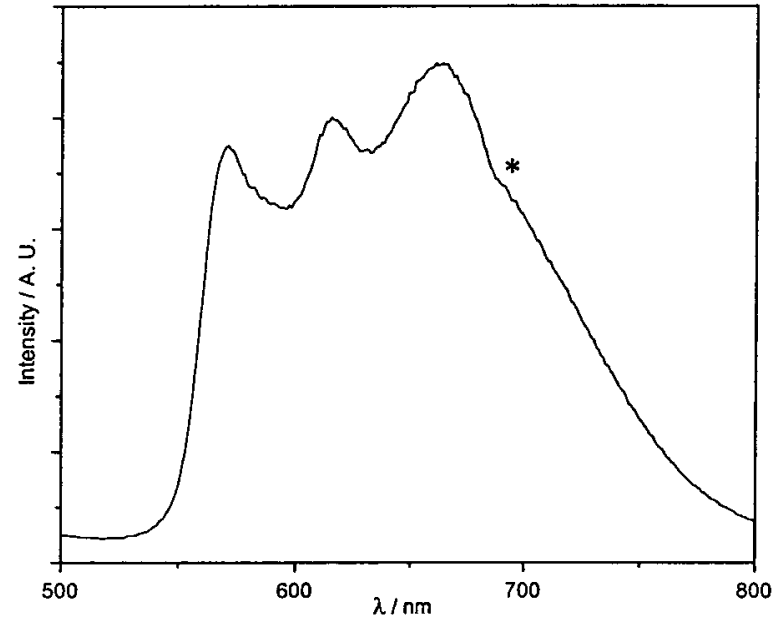

Figure 5. Solid-state emission spectrum of $\mathbf{4 a}\left(\lambda_{\mathrm{ex}}=380\right.$ $\mathrm{nm}$ ) at $298 \mathrm{~K}$ (asterisk denotes instrumental artifact).

The solid-state emission spectrum of $\mathbf{4 a}$ at $298 \mathrm{~K}$ (Figure 5) contains three bands at $\lambda_{\max } 571,617$, and $664(\max ) \mathrm{nm}$, all of which undergo nonexponential decay. Similarly, complexes $\mathbf{1}-\mathbf{3 a}$ display multiple emissions (including low-energy bands at 651-665 nm) in crystalline form at $298 \mathrm{~K}$, and these are generally red-shifted from the solution-state emission (Table 5). This can be attributed to excimeric emission arising from weak $\pi-\pi$ stacking of square planar $\mathrm{Pt}(\mathrm{II})$ species 
in the solid state, ${ }^{33}$ since these interactions are evident from the crystal structures of $\mathbf{1 a}$ (bottom of Figure 1 ) and $\mathbf{4 a}$ (Figure 3). Blue-shifts in emission maxima are exhibited upon cooling to $77 \mathrm{~K}$. For example, the solid emission of 4 a at $77 \mathrm{~K}$ shows bands at $\lambda_{\max } 579$ (sh 603, $630,659,691) \mathrm{nm}$, which are higher in energy than the $664 \mathrm{~nm}$ emission at $298 \mathrm{~K}$. These sol id-state emissions are proposed to originate from ${ }^{3} \mathrm{MLCT}$ excited states.

Complexes with Bzqn and Phpy Ligands. The UV-vis absorption spectra of $\mathbf{2 - \mathbf { 4 b }}$ in $\mathrm{CH}_{2} \mathrm{Cl}_{2}$ are similar to that for $\mathbf{1 b}$ and the bis(cyclometalated) derivative $\left[\mathrm{Pt}(\mathrm{Bzqn})_{2}\right] .^{32} \mathrm{H}$ igh-energy absorption bands with $\lambda_{\max }<390 \mathrm{~nm}$ are ascribed to ${ }^{11 \mathrm{~L}}$ transitions. The moderately intense bands at $\lambda_{\max } 410-421 \mathrm{~nm}$ and the significantly weaker low-energy absorptions $\left(\lambda_{\max }>450\right.$ $\mathrm{nm}$ ) are assigned to ${ }^{1} \mathrm{MLCT}$ and ${ }^{3} \mathrm{MLCT}$ transitions, respectively.

In contrast to the Thpy complexes, a number of Bzqn derivatives are nonemissive in fluid solution. At room temperature, only the $\mu$-pz species $\mathbf{2} \mathbf{b}$ is luminescent and displays structured emission at 497, 535, and 588 (sh) $\mathrm{nm}\left(\tau_{\mathrm{o}}=1.6 \mu \mathrm{s}, \phi_{\mathrm{o}}=9.9 \times 10^{-3}\right)$. A blue-shifted 77 $\mathrm{K}$ luminescence at 486, 522, and $560 \mathrm{~nm}$ is observed, and we tentatively assign the emission as mixed ${ }^{3} \mathrm{~L} / \beta_{-}$ MLCT. The $\mu$-aza complex $\mathbf{3 b}$ shows structured emission at $77 \mathrm{~K}$ only, while the $\mu$-bzim trimer $\mathbf{4 b}$ is nonemissive at 298 and $77 \mathrm{~K}$ in solution. The solid-state emissions of $\mathbf{1 - 3 b}$ are structured at $298 \mathrm{~K}$, and blue-shifts are again observed upon cooling to $77 \mathrm{~K}$.

The UV-vis absorption spectrum of the previously described $\mathbf{1} \mathbf{c}^{20}$ resembles those of $\mathbf{2} \mathbf{c}$ and $\mathbf{3 c}$ bearing the Phpy ligand, where ${ }^{1} \mathrm{~L},{ }^{1} \mathrm{MLCT}$, and ${ }^{3} \mathrm{MLCT}$ transitions $\left(\lambda<370, \lambda_{\max }=406-410\right.$, and $\lambda>440 \mathrm{~nm}$, respectively $)$ can be assigned. While the precursor $\mathbf{1 c}$ is nonemissive in solution at $298 \mathrm{~K}, \mathbf{2 c}$ and $\mathbf{3 c}$ exhibit structured emission at $\lambda_{\max } 487-556$ (sh 600) $\mathrm{nm}$. A longer lifetime and greater quantum yield are again detected for the $\mu$-pz complex $\mathbf{2 c}\left(\tau_{\mathrm{o}}=1.3 \mu \mathrm{s}, \phi_{\mathrm{o}}=0.039\right)$ compared to the $\mu$-aza dimer $3 \mathrm{c}\left(\tau_{\mathrm{o}}=0.29 \mu \mathrm{s}, \phi_{\mathrm{o}}=4.5 \times 10^{-4}\right)$.

Concluding Remarks. Our systematic photophysical investigation has revealed several trends. First, complexes bearing the Thpy group and in particular $\mu$-pyrazolate species display superior luminescent properties. Second, the energy of the emissions and lowest energy UV-vis bands for Thpy oligomers are generally red-shifted relative to the Bzqn and Phpy congeners. This can be illustrated by the $77 \mathrm{~K}$ emission spectra of $\mathbf{2 a}-\mathbf{c}$ in $\mathrm{CH}_{2} \mathrm{Cl}_{2}$ (Figure 6 ) and evidently reflects differences in the energies of the $\pi^{*}$ orbitals for the cyclometalating ligands. The relatively low-energy ex-

(33) Houlding, V. H.; Miskowski, V. M. Coord. Chem. Rev. 1991 $111,145$.

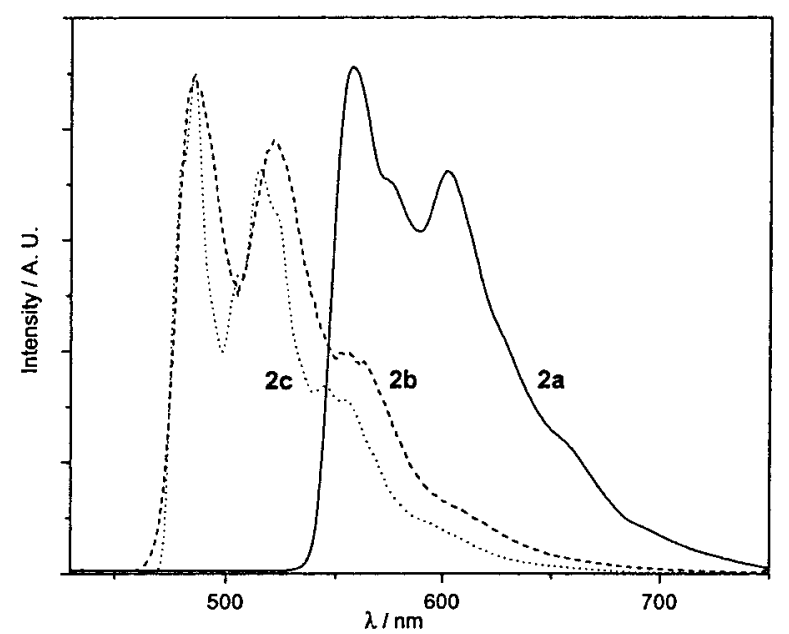

Figure 6. Normalized emission spectra of $\mathbf{2 a}-\mathbf{c}\left(\lambda_{\mathrm{ex}}=380\right.$ $\mathrm{nm}$ ) in dichloromethane at $77 \mathrm{~K}$.

cited states for Thpy derivatives result in greater energy differences between the MLCT/IL and upper-lying nonemissive MC states. Hence such complexes, in particular $[\mathrm{Pt}(\mathrm{Thpy})(\mathrm{pz})]_{2}(\mathbf{2 a})$, exhi bit improved emissi ve parameters compared to known Pt(II) binuclear complexes and are potentially interesting for photoinduced energy transfer and sensing applications.

We have described herein a simple yet efficient methodology for the selective synthesis of di- and trimeric platinum(II) macrocycles. Like the recently reported examples of molecular rectangles, ${ }^{34}$ the trimetallic molecular pockets $\mathbf{4 a}$ and $\mathbf{4 b}$ have lower symmetry than tetrametallic square anal ogues. ${ }^{35}$ While the cavity size of $\mathbf{4 a}$ and $\mathbf{4 b}$ pre-empts their employment as molecular hosts, this study has conceived a new direction for development using cyclometalated platinum(II) luminophores.

Acknowledgment. We are grateful for financial support from the University of Hong Kong and the Research Grants Council of the Hong Kong SAR, China [HKU 7298/99P].

Supporting Information Available: Tables of crystal data, atomic coordinates, calculated coordinates, anisotropic displacement parameters, and bond lengths and angles for 1a and $\mathbf{2 a}$. This material is available free of charge via the Internet at http://pubs.acs.org.

\section{OM990342H}

(34) (a) Benkstein, K. D.; Hupp, J . T.; Stern, C. L. Inorg. Chem. 1998, 37, 5404. (b) Woessner, S. M.; Helms, J . B.; Shen, Y.; Sullivan, B. P. Inorg. Chem. 1998, 37, 5406.

(35) Hunter, C. A. Angew. Chem., Int. Ed. Engl. 1995, 34, 1079. 